\title{
B.P.F.C Pure Growth Factor, PDT and LLLT for the Treatment of Severe MRONJ Non-responsive to Prolonged Antibiotic Therapy
}

\author{
Raffaello Viganò, Martina Salvatorina Murgia', Germano Orrù, Luca Vigano², Cinzia Casu \\ 1 University of Cagliari \\ 2 University of Milan
}

Funding: The author(s) received no specific funding for this work.

Potential competing interests: The author(s) declared that no potential competing interests exist.

\section{Abstract}

Drug-related osteonecrosis of the jaw (MRONJ) is a potentially serious and debilitating drug-related complication. In the literature, there are many studies that evaluate therapeutic treatments to support those already existing for MRONJ.

The aim of this work was to report a case of severe osteonecrosis, related to bisphosphonates treated with Photodynamic therapy (PDT), Low level laser therapy (LLLT) and a surgery with new platelet-rich plasma preparation. The main biological effects of LLLT are wound healing, collagen synthesis, nerve regeneration, stimulation of endorphin release and immune system modulation. PDT is a new technique for the treatment of inflammatory, infectious, ulcerative, tumor and pre-tumor lesions, able to selectively destroy the altered cells. The preparation of BioPlasma® with Pure Growth Factors, associated with the use of fibrin enriched with poor Plasma is able to provide a perfect healing. The excellent results obtained, combined with the absence of short- and long-term complications, constitute a good perspective for the outpatient use of Bio-Plasma® with Pure Growth Factors.

Therefore, LLLT and PDT associated with the use of fibrin enriched with poor Plasma, are important tools to contribute to healing and improvement of patient's quality of life.

\section{Background}

The aim of this work was to report a case of severe osteonecrosis, related to bisphosphonates (MRONJ) treated with Photodynamic therapy (PDT), Low level laser therapy (LLLT) and a delicate surgery with new platelet-rich plasma preparation. This is made without the use of anticoagulant nor any type of activator eg: bovine thrombin, calcium chloride. For this reason, it has been called "Pure", it is the B.P.F.C.® Bio-Plasma® with Pure Growth Factors (BioPlasma®) designed and developed by Dr. Raffaello Viganò (Varese, Italy 2010), to promote the healing process in patients with BRONJ ${ }^{[1]}$.

Materials and Methods 


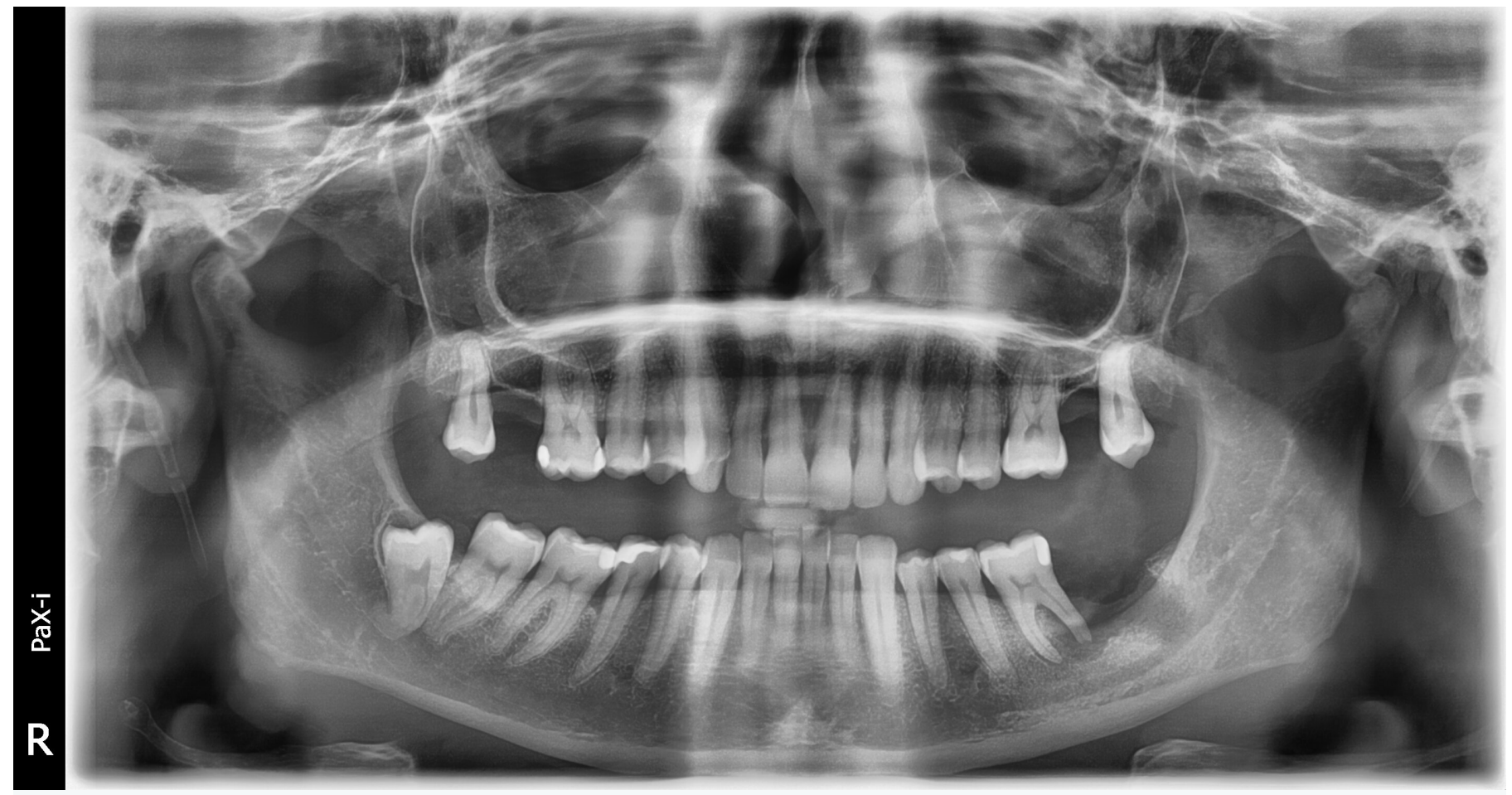

Fig. 1. OPT. 


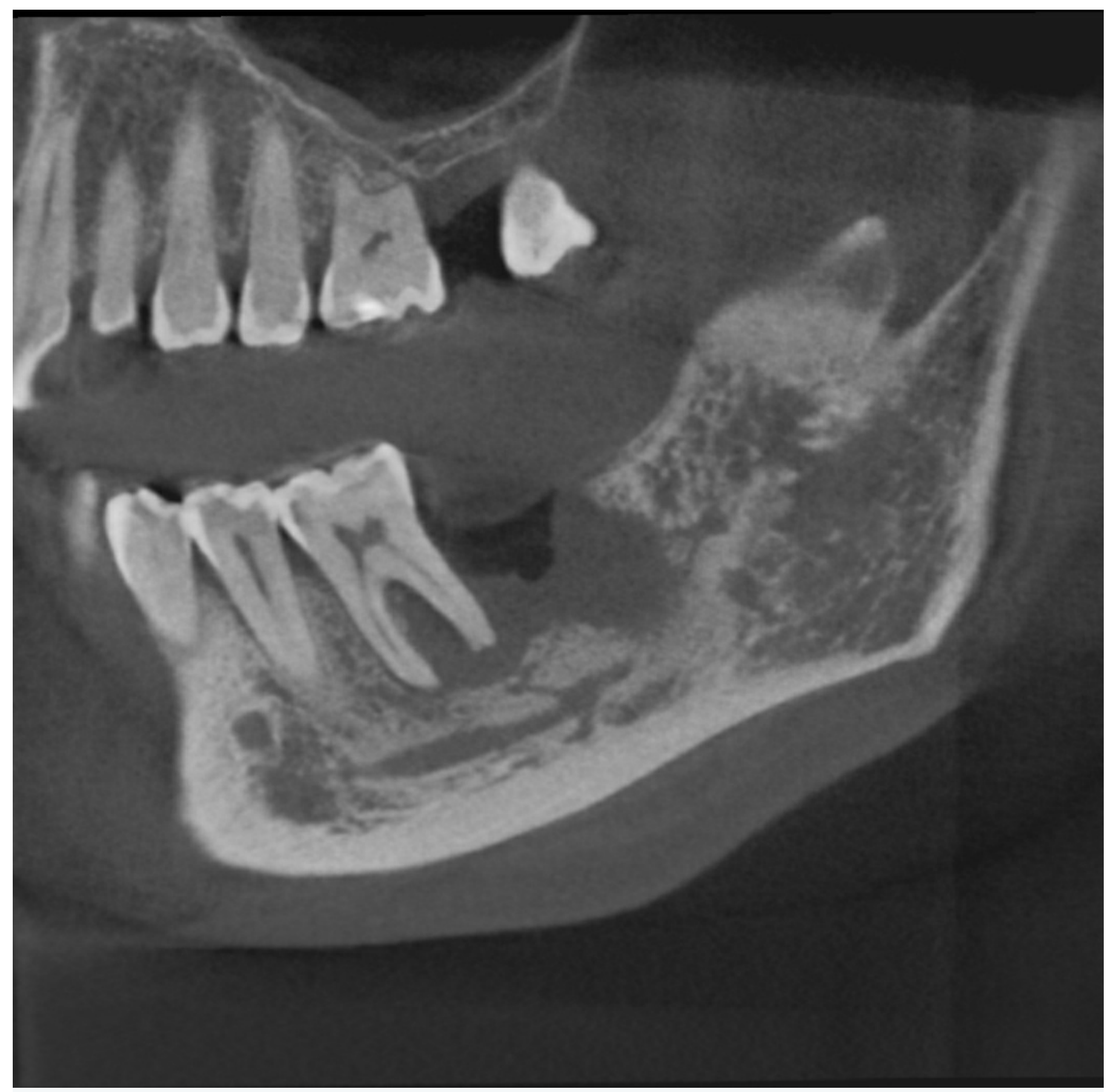

Fig. 2. Section of the affected area. 


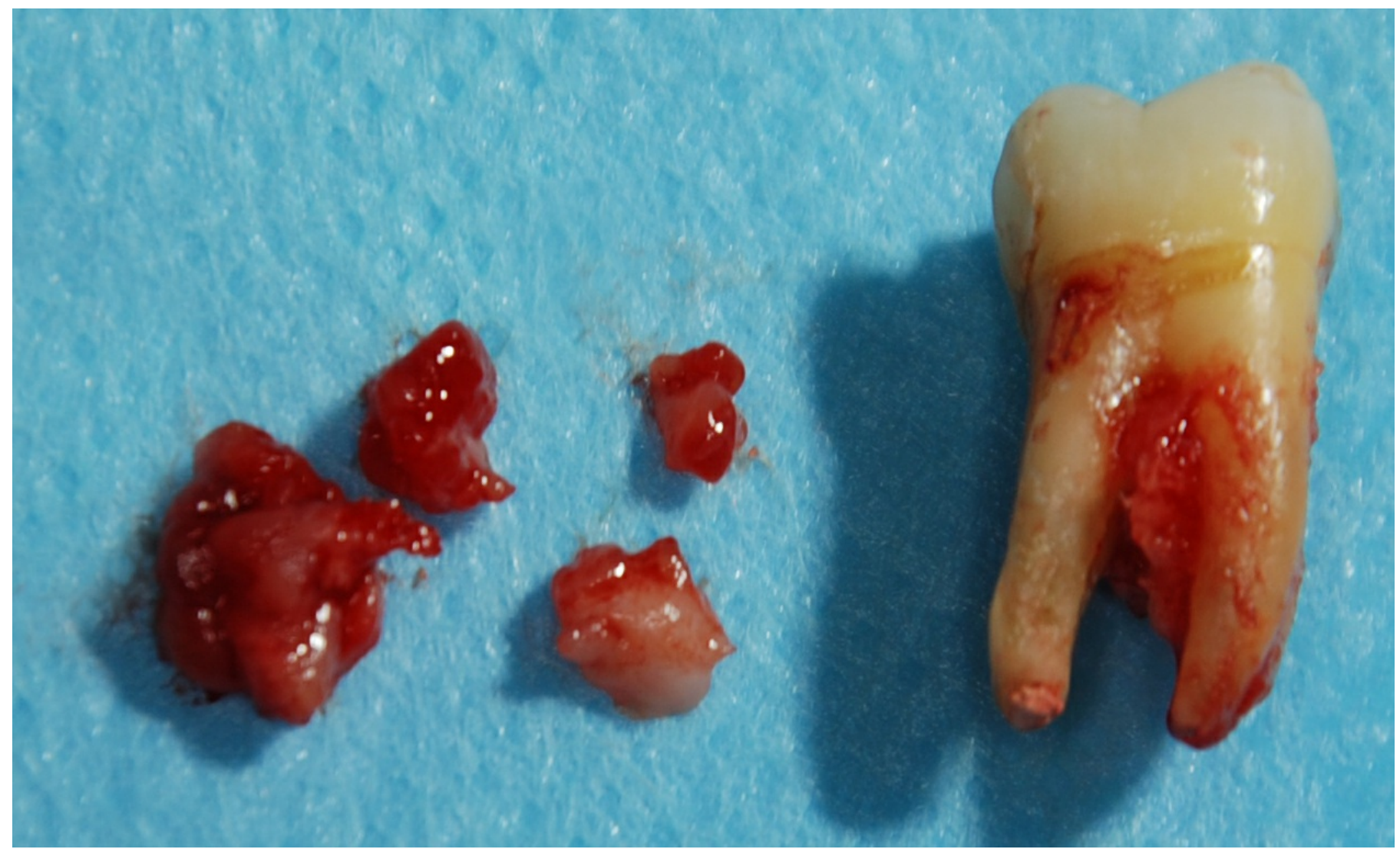

Fig. 3. Extraction of 37.

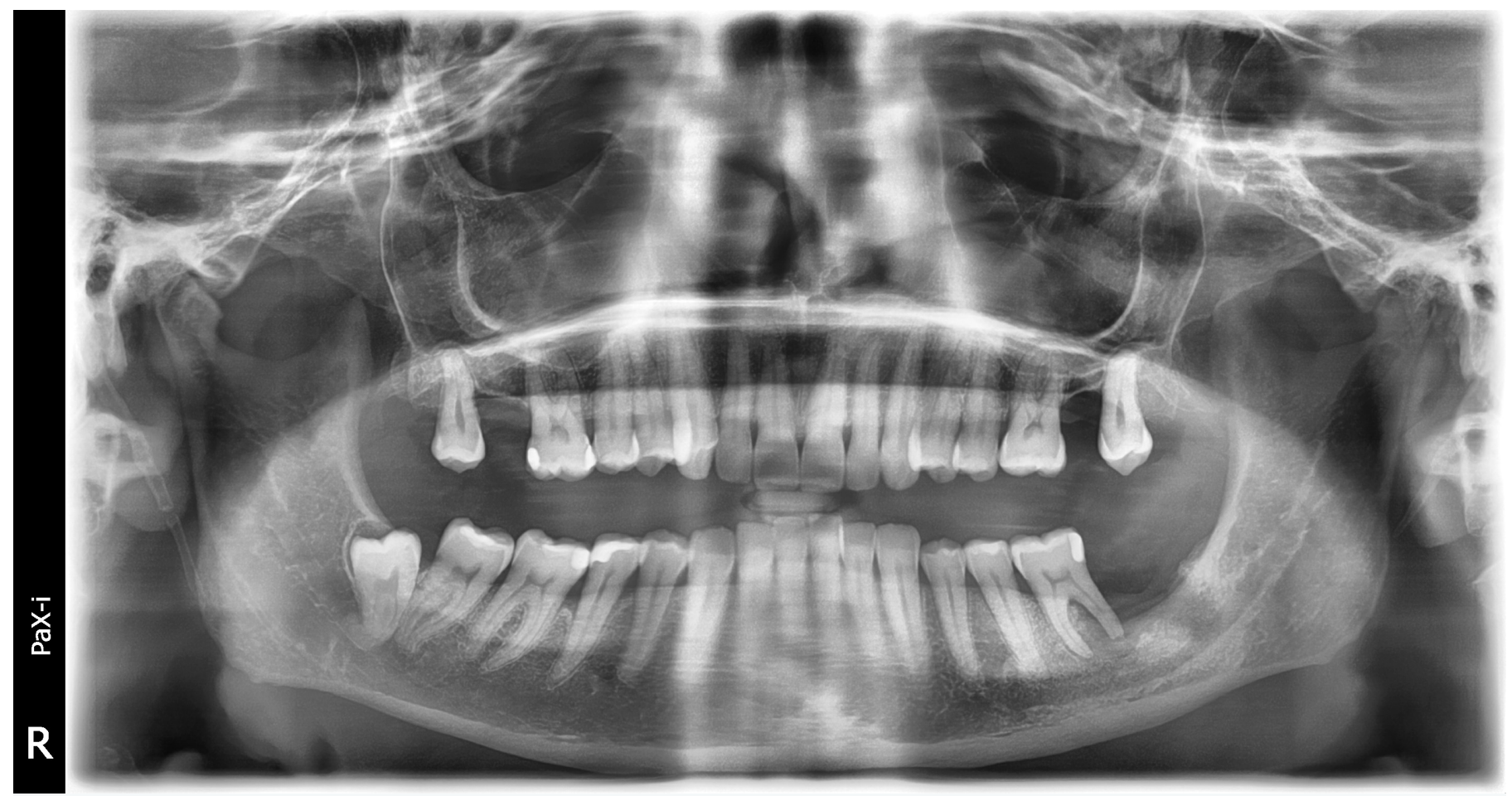

Fig. 4. OPT after antibiotic therapy and before LLLT. 


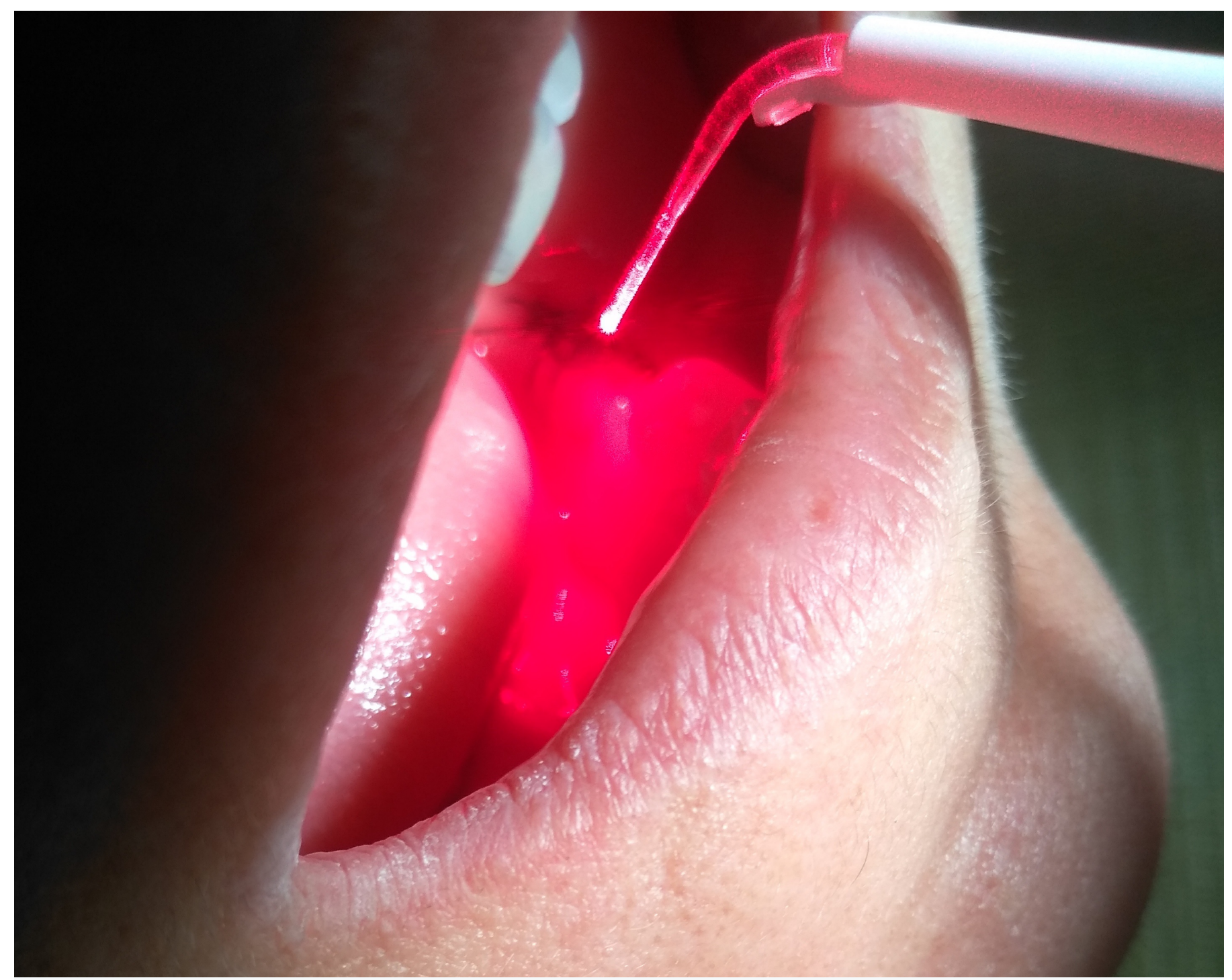

Fig. 5. LLLT. 


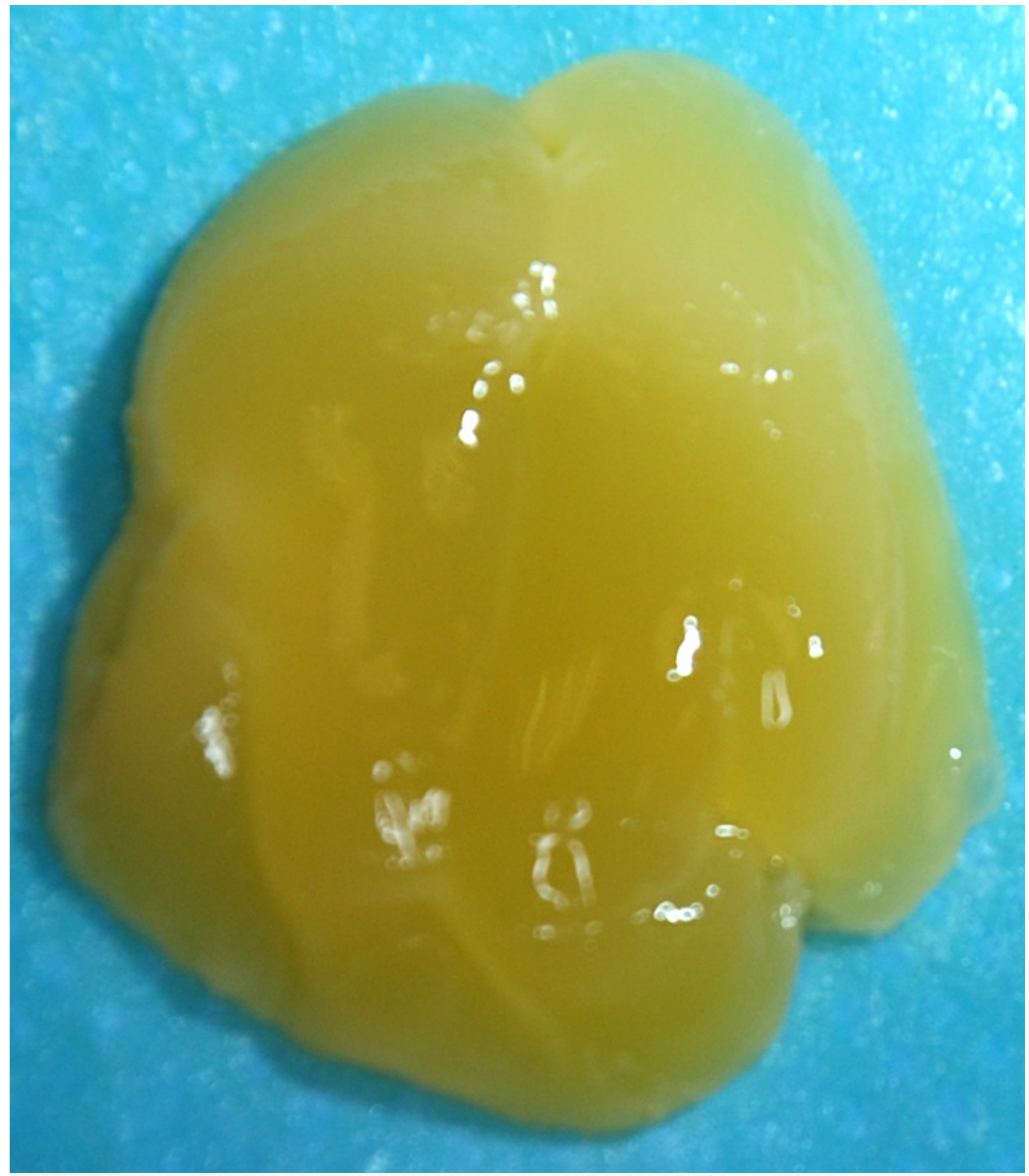

Fig. 6. Rich BioPlasma® preparation with Pure Growth Factors.

A 45-years-old female patient went to our observation with a previous diagnosis of MRONJ in the area of the elements 3.6-3.7. She reported to have been submitted to three injections of Risedronate, six months ago for a meniscus disease. Then, she received a surgery extraction of the 3.7 and she did not heal (Fig. 1-3). An antibiotic therapy (Amoxicillin+Ac.Clavulanic and Metronidazole) was prescribed, without improvements (Fig. 4). MRONJ was very near to the alveolar nerve, this would make surgery more dangerous and therefore a conservative approach is first attempted. Four PDT and LLLT sessions were performed to reduce the bacteria load and to biostimulate the bone healing (Fig. 5). PDT was performed with Methylen Blue 0,1\% and $660 \mathrm{~nm}$ diode laser (Helbo, Bredent Medical, Senden, Germany), for 5 
minutes each session. The patient was submitted to other 5 minutes of LLLT after PDT with the same laser device (100 $\mathrm{mWatt})$. After a very big improvement, we decided to treat the residual MRONJ with a regenerative surgical procedure using a rich BioPlasma® preparation with Pure Growth Factors (Fig. 6). Patient has read and signed a written consent form. The study protocol was approved by the Ethics Committee for Human Studies of the "Ospedale di Circolo" University of Varese, and was conducted in accordance with the 1975 Helsinki Declaration, amended in 2000. The phases of the study were: taking blood from the patient with Vacuette $9 \mathrm{ml}$; centrifugation and fractionation of the blood material, with elimination of red blood cells and leukocytes; creation of the membrane with a mixture of fibrin enriched with poor plasma; preparation of rich gelled plasma associated with Biomaterial and placement on the osteonecrotic site (after bone curettage and 3.6 element's avulsion); positioning of the membrane above the rich plasma; final suture with minimal exposure of the membrane, which, even if left exposed, doesn't undergo any contamination. Other two sections of PDT and LLLT, with the same previous protocol were performed.

\section{Results}

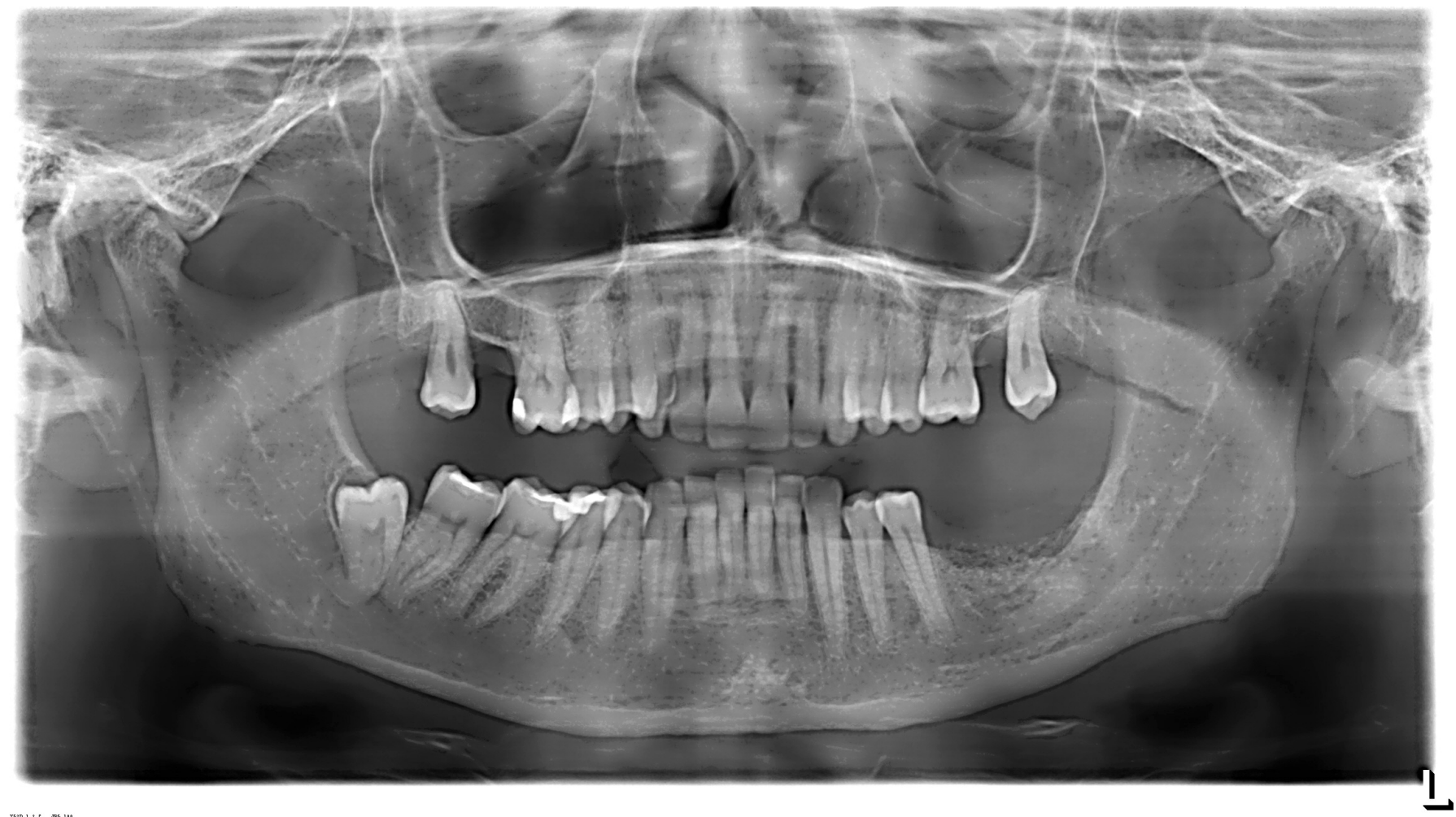

Fig. 7. OPT after 6 months.

After suture removal the patient healed. The patient was observed for the next six months without showing relapses (Fig. 7).

\section{Conclusions}


Our data showed how the preparation of Bio-Plasma ${ }^{\circledR}$ with Pure Growth Factors, inserted in the bone cavity of patients with a diagnosis of BRONJ, associated with the use of fibrin enriched with poor plasma as a suturing membrane, is able to provide perfect healing with the integrity of the hard and soft tissue after six months. PDT and LLLT could be considered very useful therapy to improve the healing process and to reduce the healing time and the invasiveness of the surgery $[2][3][4][5]$.

\section{References}

1. `Viganò R, Viganò L, Casu C. B.P.F.C. ${ }^{\circledR}$ Bio-plasma ${ }^{\circledR}$ with pure growth factors (bioPlasma ${ }^{\circledR}$ ) for the surgical treatment of patients at risk of maxillary osteonecrosis, related to bisphosphonates (BRONJ). Minerva Stomatol 2018; 67(suppl 3)p25

2. 'Casu C, Mannu C. Atypical Afta Major Healing after Photodynamic Therapy. Case Rep Dent 2017;2017:8517470

3. 'Latifyan S, Genot MT, Klatersky J. Bisphosphonate-related osteonecrosis of the jaw: a review of the potential efficacy of low-level laser therapy. Support Care Cancer 2016; 24(9):3687-93.

4. ^Minamisako MC, Ribeiro GH, Lisboa ML, Mariela Rodríguez Cordeiro M, Grando LJ. Medication-Related Osteonecrosis of Jaws: A Low-Level Laser Therapy and Antimicrobial Photodynamic Therapy Case Approach. Case Rep Dent. 2016;2016:6267406.

5. `Magalhães IA, Forte CPF, Viana TSA, Teófilo CR, Lima Verde RMB, Magalhães DP, Praxedes Neto RAL, Lima RA, Dantas TS. Photobiomodulation and antimicrobial photodynamic therapy as adjunct in the treatment and prevention of osteoradionecrosis of the jaws: A case report. Photodiagnosis Photodyn Ther. 2020 Sep;31:101959. 